\title{
Bolus Transition During Oropharyngeal Swallowing after Unilateral Cortical Stroke
}

\author{
Hyeju Han', Taeok Park², Byung-Mo Oh³, Han Gil Seo ${ }^{3}$ Youngsun Kim ${ }^{1}$ \\ ${ }^{1}$ Communication Sciences and Disorders, Ohio University, Athens, OH, USA; ${ }^{2}$ Communication Sciences and Disorders, Illinois State University, \\ Normal, IL, USA; ${ }^{3}$ Department of Rehabilitation Medicine, Seoul National University College of Medicine, Seoul, Korea
}

Purpose: Bolus transition during swallowing represents neuromuscular coordination between oropharyngeal structures and bolus movement. The purpose of this study was to examine the difference between stroke patients with left and right hemisphere lesion in terms of oral and pharyngeal transitions using oral transit time (OTT), pharyngeal transit time (PTT), and duration of upper esophageal sphincter opening (DUESO) during swallowing.

Methods: Thirty-four unilateral cortical stroke patients were divided into two groups depending on the hemispheric site of the lesion. Means and standard deviations of OTT, PTT, and DUESO were measured on $2 \mathrm{~mL}$ and $5 \mathrm{~mL}$ of thin liquid swallows from videofluoroscopic swallowing examinations (VFSES). Statistical comparison was performed by repeated measurements of analysis of variance (ANOVA). Significance level was set at $<0.025$.

Results: In OTT, PTT, and DUESO, there was no significant difference between left and right hemispheric stroke groups. There was a significant bolus volume effect on OTT and DUESO.

Conclusions: The site of the lesion may not affect the transition of bolus during oropharyngeal swallowing. Clinicians should not only examine physiological swallowing disorders of individual patients regardless of hemispheric site of the lesion, but also investigate various volumes during VFSEs in order to find the proper diet recommendation.

Keywords: Bolus transition, Oral stage, Pharyngeal stage, Swallowing, Unilateral stroke

\section{INTRODUCTION}

Swallowing is a process that transports food and liquid from the mouth to the stomach safely without the bolus entering the airway, or residue in the oral cavity and pharynx $[1,2]$. The swallowing process is controlled by the central control mechanism, which involves the cerebral cortex and the brainstem. Appropriate neurogenic sensorimotor control and movements of oropharyngeal structures can lead to smooth and safe bolus transition from the lips to the upper esophageal sphincter (UES) $[1,3,4]$.

Previous brain-imaging and/or clinical studies indicated that the cerebral cortex contribute to neuromuscular controls of not only voluntary initiation of swallowing, but also reflexive pharyngeal swallowing [5-9]. The brain-imaging studies have investigated cortical function in the swallowing of healthy adults in terms of two aspects: activated sites on the cortex during swallowing and hemispheric dominance. Researchers identified activations on the various cortical areas during swallowing. The commonly reported areas are the primary sensory/motor cortices, insula, prefrontal region, and

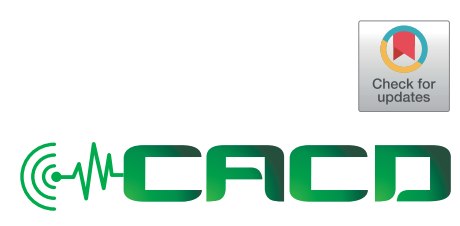

Received: September 22, 2018 Revision: December 12, 2018 Accepted: December 19, 2018

Correspondence:

Youngsun Kim

Communication Sciences and Disorders, Grover Center W218, Ohio University, Athens, OH 45701-2979, USA

Tel: $+740-597-1286$

Fax: +740-593-0287

E-mail:kimy2@ohio.edu

C 2018 The Korean Association of SpeechLanguage Pathologists

This is an Open Access article distributed under the terms of the Creative Commons Attribution NonCommercial License (http://creativecommons.org/ licenses/by-nc/4.0/) which permits unrestricted noncommercial use, distribution, and reproduction in any medium, provided the original work is properly cited. 
anterior cingulate $[6,8,10]$.

In regard to hemispheric dominance, Hamdy et al. [11] reported that electromyographic response in the mylohyoid muscle, representing muscle movement in the oral stage, was evoked by both hemispheres symmetrically, whereas electromyographic response in the pharynx and esophagus was evoked by either the left or right hemisphere without a consistent pattern among subjects. On the other hand, Kern et al. [8] identified a pattern in the relationship between the hemispheres and swallowing tasks. For voluntary initiation of swallowing, the primary sensory/motor cortices on the right hemisphere were more activated, whereas for reflexive pharyngeal swallowing, those on the left hemisphere were activated. Although the brain-imaging studies have tried to identify the dominant hemisphere for swallowing, these studies did not investigate the consistent relationship between hemispheric dominance and the bolus transition related to swallowing stages.

The relationship between the hemispheric site of the lesion and swallowing problems in each stage has been investigated in clinical research on stroke patients. Robbins et al. [9] reported that the left hemisphere stroke patients were characterized by difficulties in the oral stage, whereas the right hemisphere stroke patients showed more swallowing difficulties in the pharyngeal stage. Some clinical studies reported that there was no significant relationship between the hemispheric site of the lesion and swallowing problems in each stage [4,5,12-14]. Although some studies identified the relationship between the hemispheric site of the lesion and swallowing stage problems, there was no consistent pattern among studies. Therefore, more research is needed to identify whether each hemisphere specifically involves the oral stage, the pharyngeal stage, or both.

Temporal measurements using videofluoroscopic swallowing examination (VFSE) are useful to understand swallowing physiology [3,15]. With bolus transit times, clinicians can examine how oropharyngeal structures, such as the tongue, hyoid, larynx, pharynx, and UES, coordinate bolus movement. Previous studies have shown that the oral and pharyngeal transition of the bolus represents the integrity of physiological events in each stage [1,3,4]. In the oral stage, a bolus is formed and propelled into the pharynx by adequate coordination and pressure of the tongue. When the bolus reaches the anterior faucial pillars, or when the bolus passes the ramus of the mandible in a VFSE, the pharyngeal stage is triggered reflexively $[3,4]$. The pharyngeal stage includes safe bolus transition without the bolus entering the nasopharynx or the airway, and without residue in the pharynx. This safe transition requires sufficient and timely hyolaryngeal excursion, pharyngeal peristalsis, and UES opening [3,4]. The bolus transition in each swallowing stage is accomplished through the sequential physiological events during normal swallowing. However, impaired sensorimotor function of oropharyngeal structures results in prolonged oral transit time (OTT) and pharyngeal transit time (PTT) or in decreased duration of UES opening (DUESO) [3,16-19].

This study aimed to examine the difference between left hemisphere stroke (LHS) and right hemisphere stroke (RHS) patients regarding to oral and pharyngeal transitions. Bolus transition in each swallowing stage can be explained by three temporal measurements (e.g., OTT, PTT, and DUESO). Also, comparing quantitative data between LHS and RHS patients can provide the relationship between bolus transition in each stage and the hemispheric site of the lesion. The findings of this study would be helpful for clinicians to evaluate and treat unilateral stroke patients with swallowing disorders.

\section{METHODS}

\section{Subjects}

Videofluoroscopic swallowing examinations (VFSEs) data were collected at Seoul National University Medical Center, Seoul, Korea. There were 50 unilateral stroke patients who were referred for a VFSE from January, 2010 to July, 2014. Among these patients, 34 patients were selected from the following inclusion criteria: (1) no previous history of a stroke, (2) a completed swallowing examination, and (3) confirmed site of lesion using Computerized Tomography (CT) scans and/or Magnetic Resonance Imaging (MRI). Thirty-four patients in this investigation had middle cerebral artery (MCA) infarction. The patients were ranged in age from 41 to 91 years with a mean age of 69.53 years $(S D=10.89)$. They were divided into two groups according to the hemispheric site of the lesion. Sixteen patients (11 men and 6 women) had LHS and 18 patients (13 men and 6 women) had RHS. The Ohio University Institutional Review Board (IRB) approved this study.

\section{VFSE Procedure}

VFSEs data were collected on stroke patients. Each subject was seated upright in a wheelchair or stretcher chair for the examination. The fluoroscopic tube was focused in the lateral plane on the oral cavity (the lips anteriorly to the pharyngeal 
wall posteriorly) and the nasopharynx (superiorly) to below the UES area (inferiorly). Each subject swallowed two $2 \mathrm{~mL}$ boluses and two $5 \mathrm{~mL}$ boluses of thin liquid. The thin liquid was a mixture of water and barium sulfate powder $(35 \% \mathrm{w} / \mathrm{v})$. Each subject was instructed by the clinician to swallow after putting the liquid in his/her mouth by spoon.

Frame-by-frame images (30 frames per secord) were acquired to digitize imaging files using a computer-based image processing system equipped with a digital computer frame grabber board (Pegasus HD/SD Board, Grass Valley Inc., Honorine, France) and image processing software (EDIUS 4.5, Grass Valley Inc.). The X-ray voltage was set at a $40-\mathrm{kV}$ peak, which allowed the soft tissue of the pharyngeal structure to be visualized. VFSEs for stroke patients were adjusted, if aspiration appeared to place them at risk.

\section{Procedures for temporal measurements of bolus transit times}

This study focused on the three bolus transit times from the oral cavity to the upper esophageal sphincter: OTT, PTT, and DUESO. A total of 125 swallows were analyzed. VFSE clips were randomly mixed before analysis to avoid bias relevant to the site of the stroke lesion. To analyze the timing of swallowing events accurately, the investigators used slow motion frame-by-frame analysis by using Premiere Pro CS5.5 with a 100 millisecond video timer. OTT was measured in seconds from when the bolus head moves posteriorly to when the head of the bolus passed the ramus of the mandible $[17,18]$. PTT was measured in seconds from the bolus head arriving at the ramus of the mandible to the tail of bolus passing the UES [9]. Lastly, DUESO was measured in seconds from the initiation of the UES opening to the closure of the UES [9]. The reference points were used to calculate three measurements: (1) the initiation of posterior movement of the bolus head, (2) the bolus head passing the ramus of the mandible, (3) the tail of the bolus passing the UES, (4) the initiation opening of the UES, and (5) the closure of the UES. The OTT, PTT, and DUESO were calculated using these formulas: OTT $=(2)-(1)$, PTT $=(3)-(2)$, and DUESO = (5)-(4).

\section{Statistical analysis}

Repeated measurements of analysis of variance were performed to determine group and volume effects. Statistical analysis used bolus volume as within-subject variable and group of the site of the lesion as between-subject variable. Wilks Lamda was used to determine the significance for the temporal measurements. Significance level was set as $p<0.025$.

\section{RESULTS}

\section{Reliability}

Both intrajudge and interjudge reliability were compared by using the Pearson correlation coefficient. The significant level was set as $p<0.025$. For intrajudge reliability, the first judge reanalyzed selected $20 \%$ of subject's VFSE clips (eight subjects). Significant correlations between the first and second measurements were observed ( $r=0.98$ for OTT, $r=0.98$ for PTT, $r=0.96$ for DUESO; $p<0.01$ ). For interjudge reliability, the same $20 \%$ swallows were reanalyzed by the second judge, an experienced graduate clinician. Significant correlations between the judges were observed ( $r=0.96$ for OTT, $r=0.98$ for PTT, $r=0.96$ for DUESO; $p<0.01$ ).

\section{Oral transit time}

Table 1 shows mean time (in seconds) and the standard deviations of the OTTs of the two groups for $2 \mathrm{~mL}$ and $5 \mathrm{~mL}$ of thin liquids. In the OTTs, there was no significant difference between the LHS patients and RHS patients ( $p=0.45)$. However, there was a significant volume difference in the OTTs (Figure 1). The OTTs with $2 \mathrm{~mL}$ swallow was a 0.39 seconds longer oral transition of the bolus than those with the $5 \mathrm{~mL}$ swallow $(F(1,48)=$ $8.78, p<0.01)$. There was no interaction between hemispheric site of the lesion and bolus volume in the OTTs $(p=0.34)$.

\section{Pharyngeal transit time}

Table 2 shows means and standard deviations of the PTTs of the two groups for two volumes. In the PTTs, there was no significant difference between two groups ( $p=0.20)$. In addition, there was no significant volume difference $(p=0.03)$ and interaction between hemispheric site of the lesion and bolus volume $(p=0.04)$ (Figure 2).

\section{Duration of UES opening}

Table 3 shows means and standard deviations of the DUESOs of the two groups for two volumes. There was no significant difference between the LHS and RHS patients in DUESOs

Table 1. The mean and standard deviation $(S D)$ of the oral transit time of the two groups for $2 \mathrm{~mL}$ and $5 \mathrm{~mL}$ liquids

\begin{tabular}{lccc}
\hline Group & Volume $(\mathrm{mL})$ & Mean $(\mathrm{sec})$ & $S D$ \\
\hline Left & 2 & 1.23 & 0.95 \\
& 5 & 0.67 & 0.44 \\
Right & 2 & 1.20 & 0.80 \\
& 5 & 0.94 & 0.67 \\
\hline
\end{tabular}




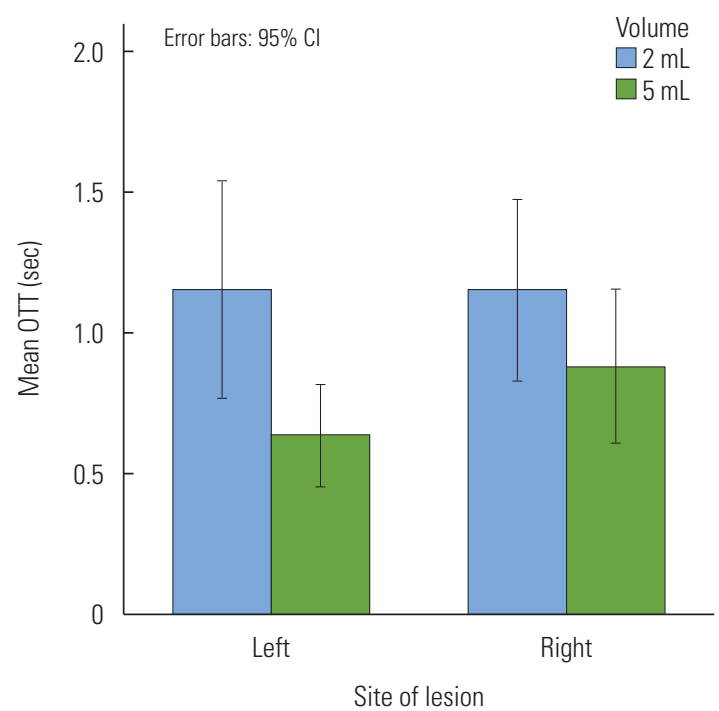

Figure 1. Mean and 95\% Confidence interval of the oral transit time (OTT) for the two groups by the $2 \mathrm{~mL}$ and $5 \mathrm{~mL}$ liquids.

Table 2. The mean and standard deviation $(S D)$ of the pharyngeal transit time of the two groups for $2 \mathrm{~mL}$ and $5 \mathrm{~mL}$ liquids

\begin{tabular}{lccc}
\hline Group & Volume $(\mathrm{mL})$ & Mean $(\mathrm{sec})$ & $S D$ \\
\hline Left & 2 & 1.39 & 1.00 \\
& 5 & 0.98 & 0.49 \\
\multirow{2}{*}{ Right } & 2 & 0.82 & 0.54 \\
& 5 & 0.85 & 0.36 \\
\hline
\end{tabular}

( $p=0.63$ ). In volume difference of the DUESOs, a significant difference was observed. The DUESOs with the $5 \mathrm{~mL}$ swallow was longer than those with $2 \mathrm{~mL}$ swallows $(F(1,52)=75.63$, $p<0.01)$. There was no interaction between hemispheric site of the lesion and bolus volume in DUESOs (Figure 3).

\section{DISCUSSION}

This study investigated the difference between LHS and RHS patients with regard to oral and pharyngeal transitions using OTT, PTT, and DUESO. In three bolus transit times, there was no significant difference between two groups. In addition, a significant volume difference in OTT and DUESO was observed.

A relationship between hemispheric dominance and bolus transit times with the $2 \mathrm{~mL}$ and $5 \mathrm{~mL}$ liquid swallows was not significant in this study. These findings indicate that there is no hemispheric difference in the oropharyngeal transition of the bolus in unilateral stroke patients. The results for OTT in this study seem to be consistent with previous studies. Dan-

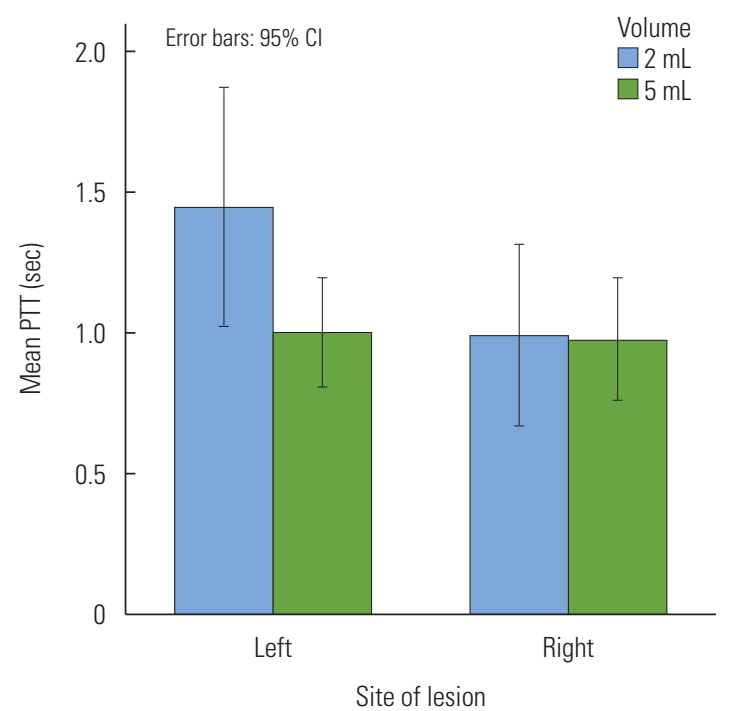

Figure 2. Mean and 95\% Confidence interval of the pharyngeal transit time (PTT) for the two groups by $2 \mathrm{~mL}$ and $5 \mathrm{~mL}$ liquids.

Table 3. The mean and standard deviation $(S D)$ of the duration of UES opening of the two groups for $2 \mathrm{~mL}$ and $5 \mathrm{~mL}$ liquids

\begin{tabular}{lccc}
\hline Group & Volume $(\mathrm{mL})$ & Mean $(\mathrm{sec})$ & $S D$ \\
\hline Left & 2 & 0.43 & 0.12 \\
& 5 & 0.53 & 0.06 \\
Right & 2 & 0.42 & 0.09 \\
& 5 & 0.52 & 0.12 \\
\hline
\end{tabular}

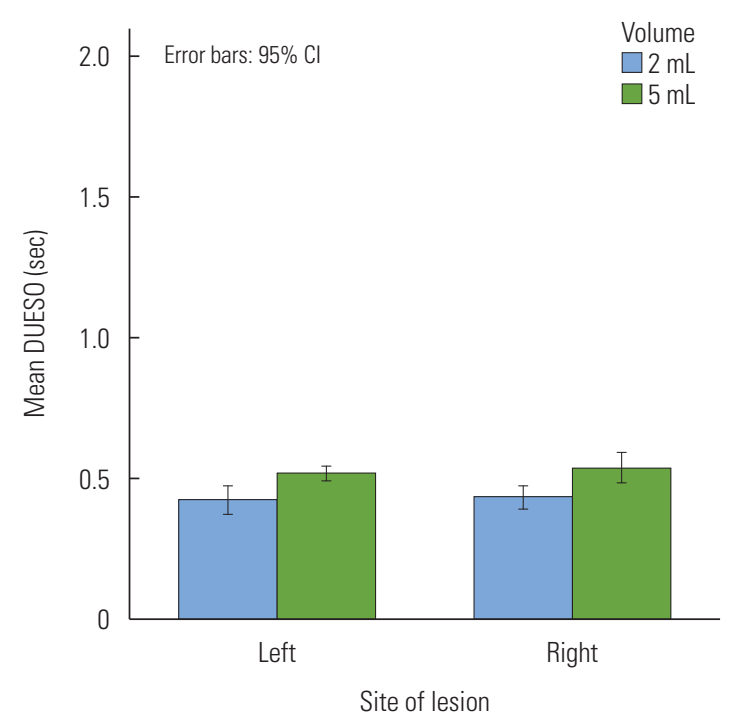

Figure 3. Mean and 95\% Confidence interval of the duration of UES opening (DUESO) for the two groups by the $2 \mathrm{~mL}$ and $5 \mathrm{~mL}$ liquids.

iels et al. [5] reported that unilateral stroke patients, regardless of the hemispheric site of the lesion, presented with swallow- 
ing difficulties in the oral stage, such as tongue pumping, delayed initiation of tongue movement, and uncoordinated initiation of oral transfer. In addition, Veis \& Logemann [4] identified reduced lingual control in both LHS and RHS patients. Also, they reported that the site of the lesion did not seem to predict specific swallowing events in either the oral and pharyngeal stage.

In addition, the results for OTT may be described by the results of cerebral cortical representation during swallowing. The several lesions, including the primary sensory/motor cortices, insular, and prefrontal cortex are involved in voluntary oral swallowing [6-8]. Thus, a cortical stroke, regardless of the lesion side, may or may not cause swallowing problems in the oral stage. According to Kim et al. [20], brain infarction in the territory supplied by the middle cerebral artery can cause damage to different anatomical structures. The unilateral stroke patients in this study had the mixed hemispheric cortical lesions in each hemisphere. In addition, oral transit time of the bolus varied to each subject. For example, three LHS patients in this study showed relatively longer OTT and 2 patients in RHS. Therefore, it is necessary to investigate the relation of specific cortical site of lesion with other physiological indicator in the oral stage of swallowing.

In the PTTs, there was also no significant difference between the two groups. The results can provide support for the results in previous studies. Johnson et al. [14] examined pharyngeal transit time in 40 stroke patients compared with 16 healthy subjects. The authors reported that there was no significant difference in pharyngeal transit time between LHS and RHS lesions. Veis \& Logemann [4] identified a delayed swallowing reflex and reduced pharyngeal peristalsis in stroke patients regardless of the lesion side.

In this study, although the result was not significant, the LHS patients tended to have longer PTTs of $2 \mathrm{~mL}$ thin liquids than of a $5 \mathrm{~mL}$ bolus in LHS and both $2 \mathrm{~mL}$ and $5 \mathrm{~mL}$ bolus in the RHS. That is, the LHS patients have longer pharyngeal transition of a $2 \mathrm{~mL}$ liquid bolus LHS patients showed difficulty to transfer the small size of the bolus in pharyngeal cavity. Small size of the bolus during pharyngeal swallowing may be required to have more effort to swallow. But LHS patients cannot manage the small size bolus effectively during pharyngeal swallowing compared to RHS patients. It is necessary to investigate the relationship between bolus size and site of lesion in stroke patients. In addition, it may be related to the distinctiveness of swallowing in stroke patients. Although subjects tended to have a dominant hemisphere for swallow- ing, the site of hemisphere with greater activation various across swallowing tasks within individuals $[6,10,11]$. In addition, Martin et al. [10] suggested that the functional lateralization of swallowing may be associated with the behavioral context for the task in which the swallowing occurs, such as a naïve saliva swallow, a voluntary saliva swallow, and a water swallow. Thus, it is essential to examine the task-specific swallowing pattern based on physiological events to understand swallowing in stroke patients.

Previous studies reported that the RHS patients showed swallowing problems in the pharyngeal stage (e.g., delayed swallowing and more residue in the pharynx) that had a high risk of aspiration or a relatively frequent occurrence of aspiration $[5,9,22]$. In this study, the occurrence of aspiration was one LHS patient and four RHS patients. Only one aspirating RHS patient (patient number 34) had a longer PTT. However, the rest of the aspirating group members did not show relatively prolonged bolus transit times as compared with a LHS patient. Thus, although more number of RHS patients showed aspiration, there was no demonstrated difference in pharyngeal bolus transit time between LHS and RHS patients.

In the DUESOs, there was no significant difference in DUESO between LHS and RHS patients. This result is consistent with those of Robbins's study [9]. Hamdy et al. [11] identified that the electromyographic response in the pharynx and esophagus was lateralized to either the left or right hemisphere without a consistent pattern among subjects. In this study, one patient in each LHS and RHS patient group had a relatively shorter DUESO than other patients and one LHS and two RHS patients had longer DUESOs than others. Therefore, the site of the lesion may not affect the transition of bolus in the UES zone.

Bolus volume differences for OTT and DUESO were observed in the present study. With increased bolus volume, OTT decreased and DUESO increased significantly. The finding of a volume difference provides support for the studies of previous research [17,23]. Rademaker et al. [23] identified that, as the bolus volume increased from $1 \mathrm{~mL}$ to $10 \mathrm{~mL}$, bolus transit times (e.g., OTT, PTT) decreased in healthy subjects. Tracy et al. [19] reported decreased OTT with increased volume through a VFSE in healthy subjects. These previous studies demonstrated that a larger volume was easier to transfer from the oral cavity to pharynx and esophagus. It is related to the notion that a small bolus with reduced sensory input requires more effort to swallow than a large bolus. Bisch et al. [24] reported that stroke patients with reduced sensory aware- 
ness had difficulty in passing a 1-2 $\mathrm{mL}$ small bolus from the mouth and into the pharynx. Thus, it is necessary to investigate strategies to enhance sensory input to trigger swallowing. Increased DUESO with increased volume is not surprising. Previous studies reported that UES opening occurred earlier and was sustained longer in accordance with increased bolus volume [25,26]. Also, Kim et al. [27] reported that larger bolus $(10 \mathrm{~mL})$ with wider maximum width of UES opening than smaller bolus ( $5 \mathrm{~mL}$ ). The results of this study found significant volume differences in each bolus transit time in unilateral stroke patients. Therefore, clinicians should pay attention to whether unilateral stroke patients can manipulate oropharyngeal structures depending on bolus volume.

Based on the findings of this study, unilateral cortical stroke patients may have various swallowing problems throughout the entire swallowing process regardless of the side of the lesion. The patients with prolonged bolus transit times in this study showed also swallowing apraxia, premature spillage, delayed pharyngeal swallowing, and/or residue in oropharyngeal structures. These physiological disorders in unilateral stroke patients may be related to the occurrence of aspiration. In this study, one LHS and four RHS patients had aspiration. Thus, it is important to develop adequate intervention plan for each physiological disorders of swallowing.

This study showed that a stroke causes a sensory impairment and insufficient strength of the oropharyngeal structures, thereby resulting in longer transit time of the bolus. In addition, some stroke patients in this study showed impaired bolus formation resulting from incoordination of the tongue. These patients took a long time to transfer the bolus in the oral stage. For the patients who showed longer transit times with $2 \mathrm{~mL}$ swallows in this study, a larger volume may help them improve sensation in the oropharynx. Therefore, it is essential for clinicians to examine physiological disorders of each patient regardless of hemispheric lateralization.

This study has several limitations. A small number of unilateral stroke patients participated. As previous VFSE data were used, some data were missing. Thus, the significant findings of this study should be interpreted carefully. The bolus volume differences in the bolus transit times were observed between $2 \mathrm{~mL}$ and $5 \mathrm{~mL}$ of thin liquid. Future studies should examine a relationship between bolus transit times and hemispheric dominance with various bolus volumes and/or consistencies. In addition, a number of unilateral stroke patients in this study showed delayed pharyngeal swallowing. Thus, further study is needed to identify a relationship between de- layed pharyngeal swallowing and the hemispheric site of the unilateral stroke lesion by measuring parameters indicating delayed pharyngeal swallowing.

\section{REFERENCES}

1. Dodds WJ, Logemann JA, Stewart ET. Radiologic assessment of abnormal oral and pharyngeal phases of swallowing. AJR Am J Roentgenol. 1989;154:965-974.

2. Mann G, Hankey GJ, Cameron D. Swallowing function after stroke: prognosis and prognostic factors at 6 months. Stroke. 1999;30:744748.

3. Logemann JA. Evaluation and treatment of swallowing disorders. 2nd ed. Austin, TX:Pro-ed; 1998.

4. Veis SL, Logemann JA. Swallowing disorders in person with cerebrovascular accident. Am J Phys Med Rehabil. 1985;66:372-375.

5. Daniels SK, Foundas AL, Iglesia GC, Sullivan MA. Lesion site in unilateral stroke patients with dysphagia. J Stroke Cerebrovasc Dis. 1996;6:30-34.

6. Hamdy S, Mikulis DJ, Crawley A, Xue S, Lau H, Henry S, et al. Cortical activation during human volitional swallowing: an event-related fMRI study. Am J Phys. 1999;277:219-225.

7. Hamdy S, Rothwell JC, Aziz I, Thompson DG. Organization and reorganization of human swallowing motor cortex: implications for recovery after stroke. Clin Sci. 2001;98:151-157.

8. Kern MK, Jaradeh S, Arndorfer RC, Shaker R. Cerebral cortical representation of reflexive and volitional swallowing in humans. Am J Physiol Gastrointest Liver Physiol. 2001;280:354-360.

9. Robbins J, Levine RL, Maser A, Rosenbek JC, Kempster GB. Swallowing after unilateral stroke of the cerebral cortex. Arch Phys Med Rehabil. 1993;74:1295-1300.

10. Martin RE, Goodyear BG, Gati JS, Menon RS. Cerebral cortical representation of automatic and volitional swallowing in humans. J Neurophysiol. 2001;85:938-950.

11. Hamdy S, Aziz Q, Rothwell JC, Singh KD, Barlow J, Hughes DG, et al. The cortical topography of human swallowing musculature in healthy and disease. Nat Med. 1996;2:1217-1224.

12. Chen MY, Ott DJ, Peele VN, Gelfand DW. Oropharynx in patients with cerebrovascular disease: evaluation with videofluoroscopy. Radiology. 1999;176:641-643.

13. Irie H, Lu C. Dynamic evaluation of swallowing in patients with cerebrovascular accident. Clin Imaging. 1995;19:240-243.

14. Johnson ER, McKenzie SW, Rosenquist J, Liebermann JS, Sievers AE. Dysphagia following stroke: quantitative evaluation of pharyngeal transit times. Arch Phys Med Rehabil. 1992;73:419-423.

15. Mendell DA, Logemann JA. Temporal sequence of swallow events during the oropharyngeal swallow. J Speech Lang Hear Res. 2007; 50:2156-2171.

16. Perlman AL, Booth BM, Grayhack JP. Videofluoroscopic predictors of aspiration in patients with oropharyngeal dysphagia. Dysphagia. 1994;9:90-95.

17. Park T, Kim Y, McCullough G. Oropharyngeal transition of the bo- 
lus in post-stroke patients. Am J Phys Med Rehabil. 2013;92:320326.

18. Power ML, Hamdy S, Goulermas, JY, Tyrrell PJ, Turnbull I, Thompson DG. Predicting aspiration after hemispheric stroke from timing measures of oropharyngeal bolus flow and laryngeal closure. Dysphagia. 2009;24:257-264.

19. Tracy JF, Logemann JA, Kahrilas PJ, Jacob P, Kobara M, Krugler C. Preliminary observations on the effects of age on oropharyngeal deglutition. Dysphagia. 1989;4:90-94.

20. Kim SY, Kim TU, Hyun JK, Lee SJ. Differences in videofluoroscopic swallowing study (VFSS) findings according to the vascular territory involved in stroke. Dysphagia. 2014;29:444-449.

21. Robbins JA, Levine RL. Swallowing after unilateral stroke of the cerebral cortex: preliminary experience. Dysphagia. 1988;3:11-17.

22. Smithard DG, O'Neill PA, Martin DF, England R. Aspiration following stroke: is it related to the side of the stroke? Clin Rehabil. 1997;11:73-76.
23. Rademaker AW, Pauloski BR, Colangelo LA, Logemann JA. Age and volume effects on liquid swallowing function in normal women. J Speech Lang Hear Res. 1998;41:275-284.

24. Bisch EM, Rademaker AW, Kahrilas PJ, Lazarus CL. Pharyngeal effects of bolus volume, viscosity, and temperature in patients with dysphagia resulting from neurologic impairment and in normal subjects. J Speech Lang Hear Res. 1994;37:1041-1049.

25. Kahrilas PJ, Logemann JA. Volume accommodation during swallowing. Dysphagia. 1993;8:259-265.

26. Bulter SG, Stuart A, Castell D, Russell GB. Effects of age, gender, bolus condition, viscosity, and volume on pharyngeal and upper esophageal sphincter pressure and temporal measurements during swallowing. J Speech Lang Hear Res. 2009;52:240-253.

27. Kim Y, Park T, Oommen E, McCullough G. Upper esophageal sphincter opening during swallow in stroke survivors. Am J Phys Med Rehabil. 2015;94:734-739. 